\title{
Invariant manifolds of the Bonhoeffer-van der Pol oscillator
}

\author{
R. Benítez ${ }^{1}$, V. J. Bolós ${ }^{2}$ \\ ${ }^{1}$ Departamento de Matemáticas, \\ Centro Universitario de Plasencia, Universidad Extremadura. \\ Avda. Virgen del Puerto 2, 10600 Plasencia, Spain. \\ e-mail: rbenitez@unex.es \\ 2 Departamento de Matemáticas, \\ Facultad de Ciencias, Universidad Extremadura. \\ Avda. de Elvas s/n, 06071 Badajoz, Spain. \\ e-mail: vjbolos@unex.es
}

October 2007

\begin{abstract}
The stable and unstable manifolds of a saddle fixed point (SFP) of the Bonhoeffer-van der Pol oscillator are numerically studied. A correspondence between the existence of homoclinic tangencies (which are related to the creation or destruction of Smale horseshoes) and the chaos observed in the bifurcation diagram is described. It is observed that in the nonchaotic zones of the bifurcation diagram, there may or may not be Smale horseshoes, but there are no homoclinic tangencies.
\end{abstract}

Keywords: Bonhoeffer-van der Pol oscillator; Smale horseshoes; chaos; bifurcation; invariant manifolds.

\section{Introduction}

The Bonhoeffer van der Pol oscillator (BvP) is the non-autonomous planar system

$$
\left.\begin{array}{rl}
x^{\prime} & =x-\frac{x^{3}}{3}-y+I(t) \\
y^{\prime} & =c(x+a-b y)
\end{array}\right\},
$$

being $a, b, c$ real parameters, and $I(t)$ an external forcement. We shall consider only a periodic forcement $I(t)=A \cos (2 \pi t)$ and the specific values for the 


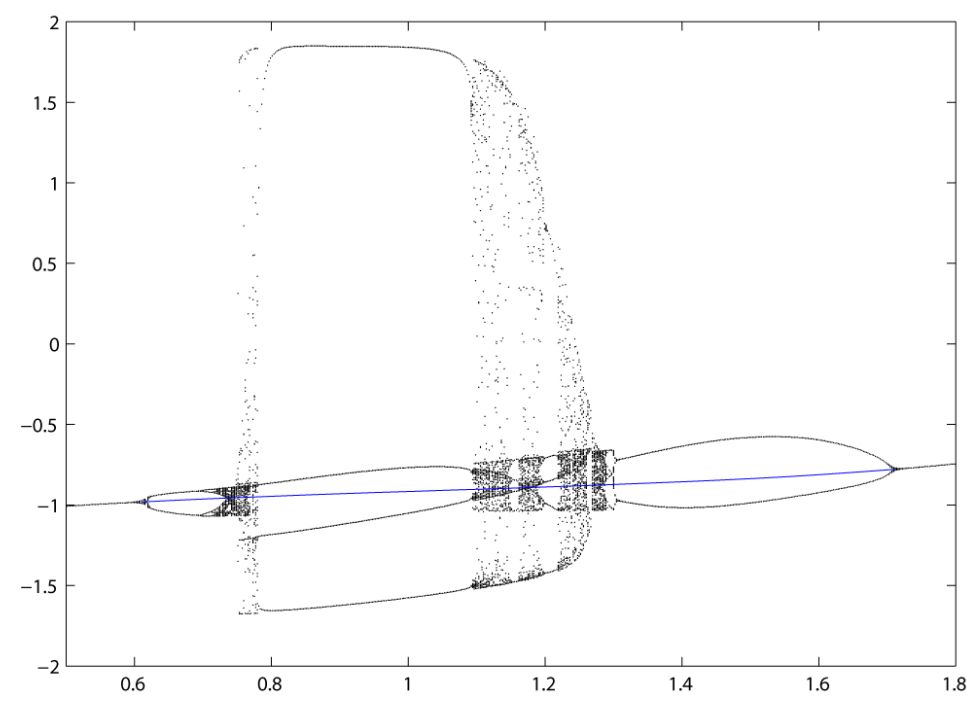

Figure 1: Bifurcation diagram for the first coordinate of the periodic points of the Poincaré map, and $0.5 \leq A \leq 1.8$. The blue line represents the position of the Saddle Fixed Point (SFP).

parameters $a=0.7, b=0.8, c=0.1$. These values were considered in [1] because of their physical and biological importance (see [2]).

In previous works [3, the existence of "horseshoe chaos" in BvP was studied analitically by means of the Melnikov method applied to an equivalent system, the Duffing-van der Pol oscillator (DvP). It was concluded that the BvP system should have Smale horseshoes. Nevertheless, the method used there can be applied only for $b>1$ and $c<1 / b$; which is not our case. In this work we will show the relation between the chaos transitions in the BvP system (1) and the creation or destruction of Smale horseshoes. Such horseshoes will be identified by the existence of homoclinic tangencies between the invariant manifolds of a saddle fixed point of the Poincaré map. With this aim we perform a numerical descriptive analysis of the stable and unstable manifolds.

\section{The bifurcation diagram}

Obtaining, for different values of the parameter $A$, the periodic fixed points of the Poincaré map (which is defined by the flow of the system on $t=1$, see [4]), a typical bifurcation diagram is found, with chaotic and non chaotic zones (see Fig. 1). Such diagram has been deeply studied in [5] and [1].

For $0 \lesssim A \lesssim 0.61$ and for $1.72 \lesssim A$, there is a unique attracting fixed point. The first bifurcation takes place in $A \approx 0.61$, where two attracting 2-periodic points appear, and a saddle fixed point (SFP) between them. Our aim is to study the invariant manifolds of such SFP. 

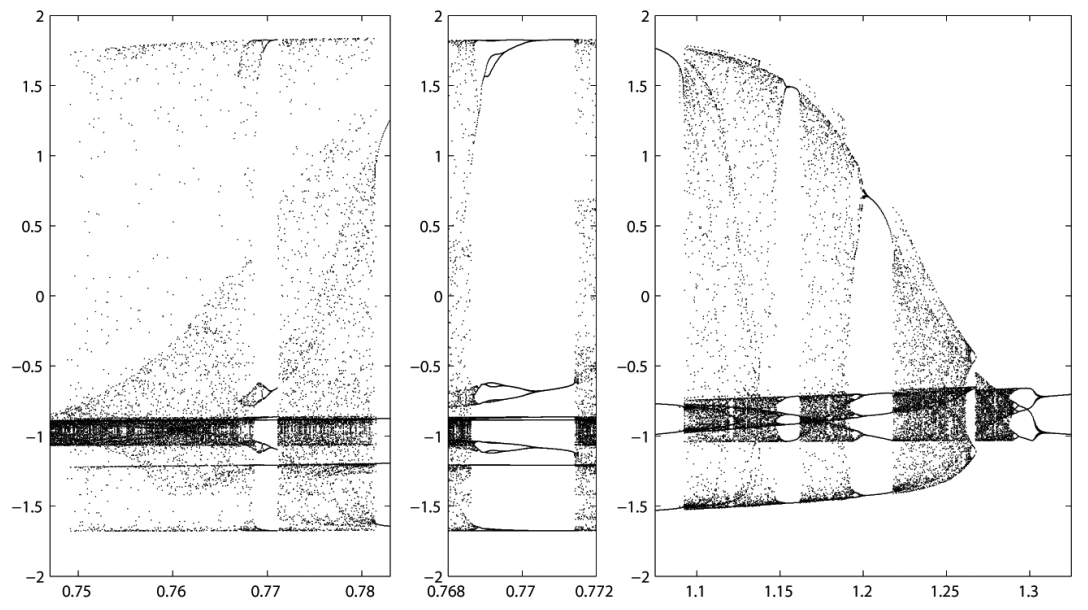

Figure 2: Some details of the bifurcation diagram of the $x$-coordinate.

For $0.61 \lesssim A \lesssim 0.735$ the bifurcation diagram is very simple, from a dynamical point of view. The attracting periodic points keep doubling its periods and forming a typical non-chaotic bifurcation diagram. Their attraction basins are perfectly separated and no fractal structures are formed.

For $0.735 \lesssim A \lesssim 1.2835$ there is a sequence of chaotic and non-chaotic zones (see Fig. 22). The largest non-chaotic zone yields for $0.782 \lesssim A \lesssim 1.092$, and there is a 4-periodic attracting point. For example, the four periodic points in the case $A=0.85$ are $x_{1} \approx(-1.6444,0.4723), x_{2} \approx(-1.1557,-0.2346)$, $x_{3} \approx(-0.8362,-0.4906), x_{4} \approx(1.8481,0.4416)$, and the image of any of them is the next one, i.e. $f\left(x_{i} \bmod 4\right)=x_{i+1} \bmod 4$.

Another event that must be mentioned is the sudden expansion of the attractor that takes place at $A \approx 0.748$; once the horseshoe chaos has begun. Shuch expansion has been deeply studied in [1] using dynamical structure functions.

For $1.2835 \lesssim A \lesssim 1.72$ there is no horseshoe chaos. In this zone there are attracting periodic points. This situation is held until $A \approx 1.72$, where the SFP vanishes and a unique attracting fixed point appears.

\section{Invariant manifolds}

As we have mentioned above, our aim is to relate the chaos transitions in the bifurcation diagram to the creation and destruction of Smale horseshoes, which we shall identify with the existence of homoclinic tangencies between the invariant manifolds. In this section we are going to set the basic definitions and the notation related to the invariant manifods; then we shall describe the structure of the invariant manifolds, depending on the value of the parameter $A$. 


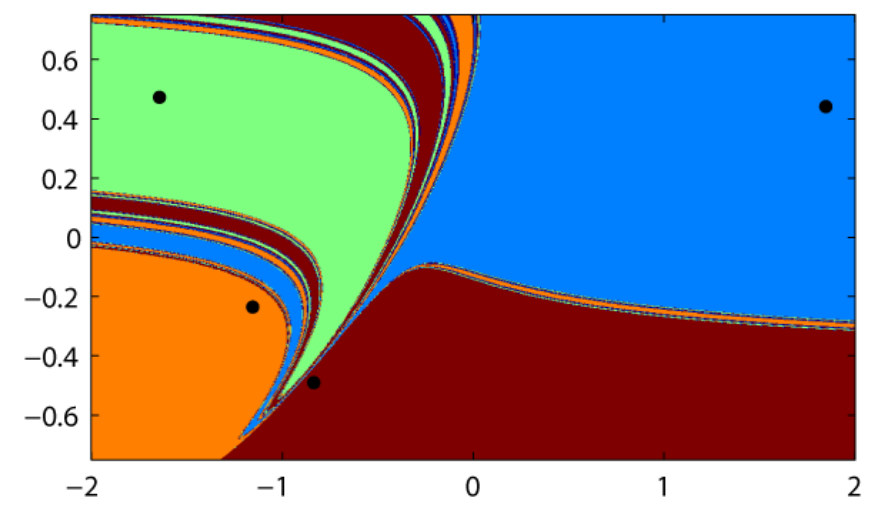

Figure 3: Attraction basins of the four attracting periodic points for $A=0.85$. For such $A$, the SFP lies approximately in $(-0.9384,-0.4146)$, which is in the boundary of the four attraction basins.

Let $x_{0}$ be a SFP of a discrete dynamical system with a two-dimensional state space, given by a continuous map $f: \mathbb{R}^{2} \rightarrow \mathbb{R}^{2}$. The stable manifold of $x_{0}, W^{s}\left(x_{0}\right)$, is defined as

$$
W^{s}\left(x_{0}\right):=\left\{x \in \mathbb{R}^{2} \quad: \quad \lim _{n \rightarrow \infty} f^{n}(x)=x_{0}\right\} .
$$

On the other hand, the unstable manifold of $x_{0}, W^{u}\left(x_{0}\right)$, is defined as

$$
W^{u}\left(x_{0}\right):=\left\{x \in \mathbb{R}^{2} \quad: \quad \lim _{n \rightarrow \infty} f^{-n}(x)=x_{0}\right\} .
$$

In this case, these invariant manifolds are one-dimensional. Moreover, it is obvious that if they intersect at one point different from the SFP, then they must intersect at an infinite set of points and Smale horseshoes are formed (see (4)).

A simple consequence of these definitions is that, in presence of several attracting fixed points, $W^{s}\left(x_{0}\right)$ is included in the boundary of the attraction basins of these points. Thus, if there are horseshoes, the attraction basins present a fractal structure (see Fig. 3).

Each invariant manifold has two branches that "arrive" at the SFP (in the case of the stable manifold) or "leave" the SFP (for the unstable manifold). More precisely, let $x \in W^{s}\left(x_{0}\right)$, then $d^{s}\left(f^{n}(x), x_{0}\right) \rightarrow 0$ as $n \rightarrow \infty$, being $d^{s}$ the arclength distance defined on $W^{s}\left(x_{0}\right)$. On the other hand, if $x \in W^{u}\left(x_{0}\right)$, then $d^{u}\left(f^{n}(x), x_{0}\right) \rightarrow \infty$, being $d^{u}$ the arclength distance defined on $W^{u}\left(x_{0}\right)$ (see [6]).

In our case, the vector field $f$ is given by the Poincaré map defined by the flow of the system (1) on $t=1$. This map has a unique SFP which appears with the first bifurcation at $A \approx 0.61$ and vanishes with the last bifurcation, 

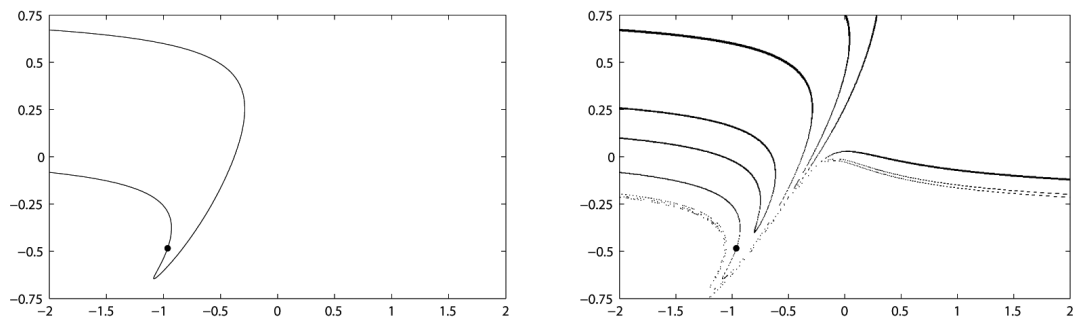

Figure 4: Stable manifold for $A=0.70$. Left: branches of the manifold plotted with the inverse method, before they get out of range. Right: stable manifold plotted with the method of "convergent points". The SFP is marked with a black circle.

approximately at $A \approx 1.72$ (see Fig. 1). Therefore we are going to focus on the values $0.61 \lesssim A \lesssim 1.72$, which are the ones for which the invariant manifolds exist.

\subsection{The stable manifold}

The simplest method for plotting the stable manifold is the well known "inverse method", which consists in plotting the unstable manifold of the inverse of the Poincaré map.

With this method, taking into account the machine precision we are using, we obtain that the branches of the stable manifold reach a numerical infinity at a finite time. This "out of range" takes place only for the $x$ coordinate, due to the cubic term of (1). Nevertheless it is important to remark that, in theory, no point on the stable manifold can go to infinity at a finite time.

Because of this behaviour, it is not possible to draw numerically the whole manifold using the inverse method. In fact, it only works until the branches are out of range (Fig. 4 left).

Recently, new algorithms for plotting stable manifolds without computing the inverse have been developed (see [6, 7, 8]). However this methods cannot be used here because they require that the manifolds are sufficiently bounded.

To avoid these problems, in order to plot the stable manifold, we use another complementary method which consists in finding the points that, after a given number of iterations, lie near the SFP (Fig. 4 right). Nevertheless this method is computationally more expensive. For more details refer to Section 5 .

\subsection{The unstable manifold}

The unstable manifold remains in a bounded region close to the SFP. However, the branches of the manifold present some folds that, at first sight, look like vertices, difficulting its study. 


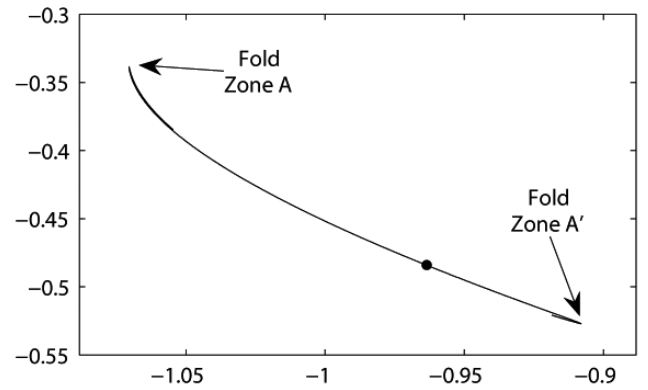

Figure 5: Unstable manifold for $A=0.70$. The SFP is marked with a black circle.

For example, for $A=0.70$, the SFP is at $(-0.9635,-0.4841)$, approximately. One branch arrives to the zone near $(-1.0704,-0.3385)$ where it folds (fold zone A). Other fold zones that have to be mentioned are near the points $(-1.0545,-0.3848)$ (fold zone B) and $(-1.0701,-0.341)$ (fold zone C). Since our Poincaré map is orientation reversing, the other branch of the manifold has an analogous structure, presenting the fold zones A', B', and C', as the images of the fold zones A, B, and C. The branches are "trapped" between the fold zones B-C and B'-C' respectively (see Figures 5,6 and 7).

If the parameter $A$ is increased, the fold zones B, C, B', C' approach the SFP (Figures 8, 9). When these fold zones arrive near the SFP, the unstable and stable manifolds will intersect, causing the first set of Smale horseshoes. For example, if $A=0.735$, these four fold zones are very close to the SFP (Fig. 10). For $A=0.74$ the first set of horseshoes has been created (Fig. 11).

As we have mentioned in Section 2, for $A \approx 0.748$ there is a sudden expansion of the size of the attractor, and consequently of the size of the unstable manifold. For example, this expansion is well described in Fig. 11 for $A=0.75$. In this case the main fold zones are near the points $(1.7458,0.2452),(-1.6781,0.4752)$, $(-1.2212,-0.2360)$, and $(-0.8721,-0.5232)$, which are the zones of the bifurcation diagram with greater density of points.

In the non-chaotic big zone $0.782 \lesssim A \lesssim 1.092$ there are four attracting points of period 4 . These points are near the main fold zones as we can see for $A=0.85$ in Fig. 11 .

\section{Smale horseshoes}

For $0.61 \lesssim A \lesssim 0.735$ there are no intersections between the stable and unstable manifolds. So there is no horseshoe chaos in this region (Fig. 12).

For $A=0.735$ it is observed that several homoclinic tangencies are about to be formed. This will cause the creation of the first set of horseshoes and the first chaos transition. All the Smale horseshoes are in the region $0.735 \lesssim A \lesssim 1.2835$. 

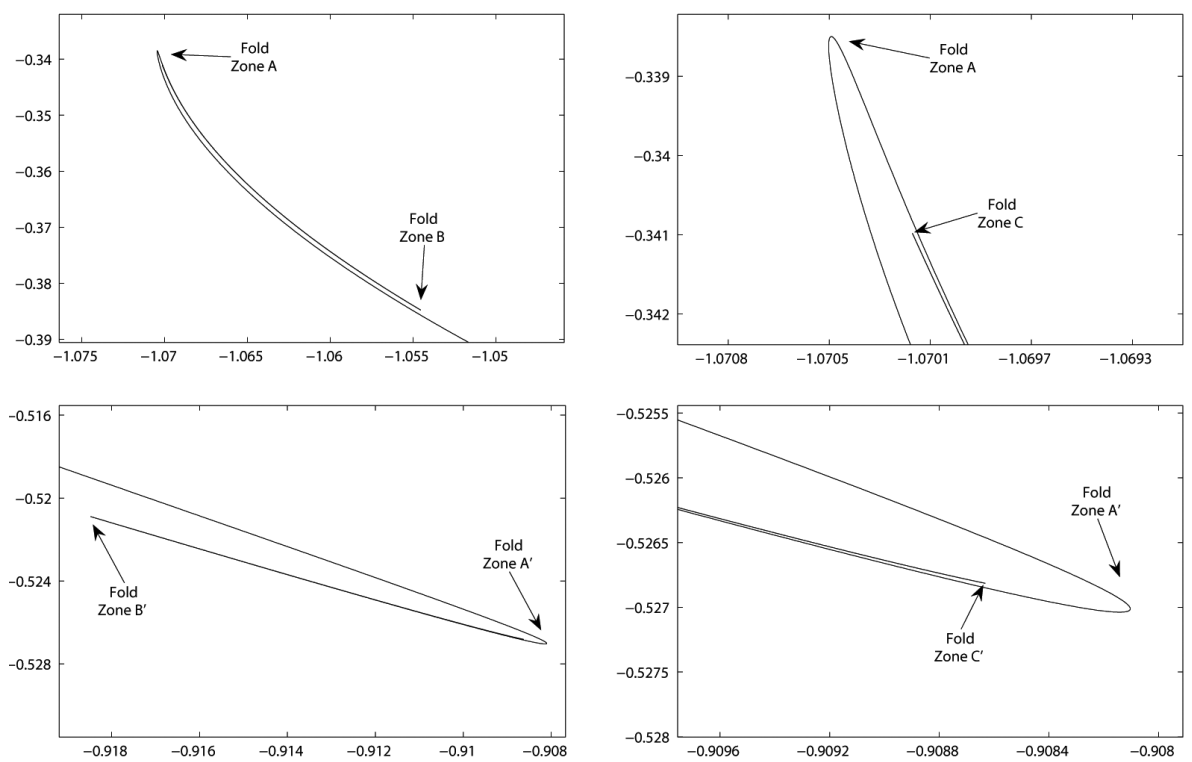

Figure 6: Details of the unstable manifold for $A=0.70$.
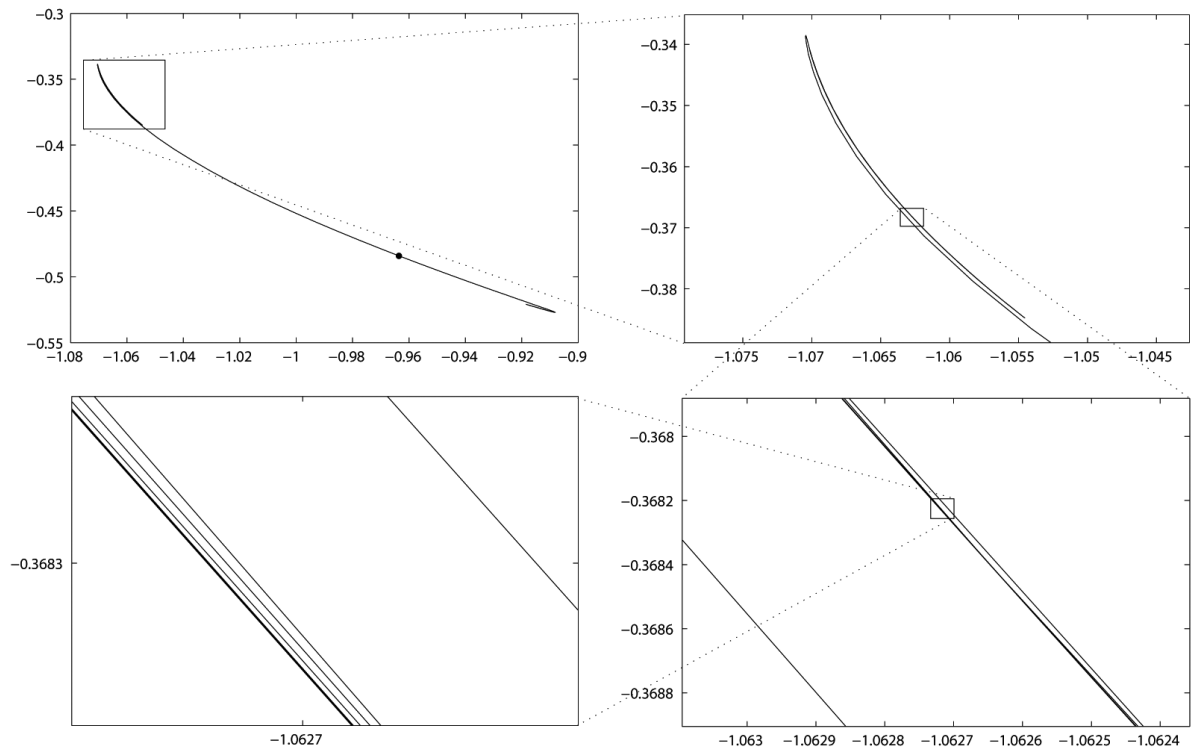

Figure 7: Details of the unstable manifold for $A=0.70$. 


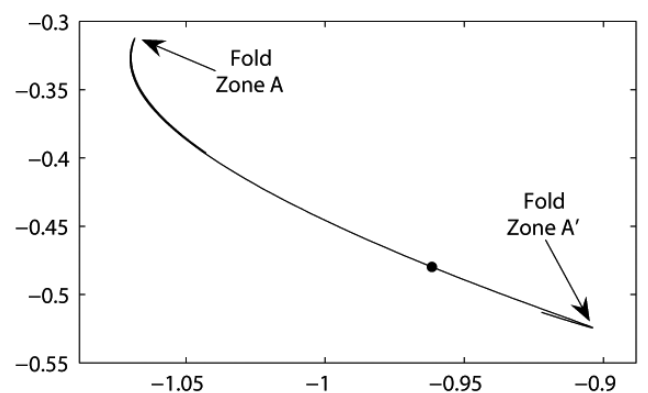

Figure 8: Unstable manifold for $A=0.71$. The SFP is marked with a black circle.
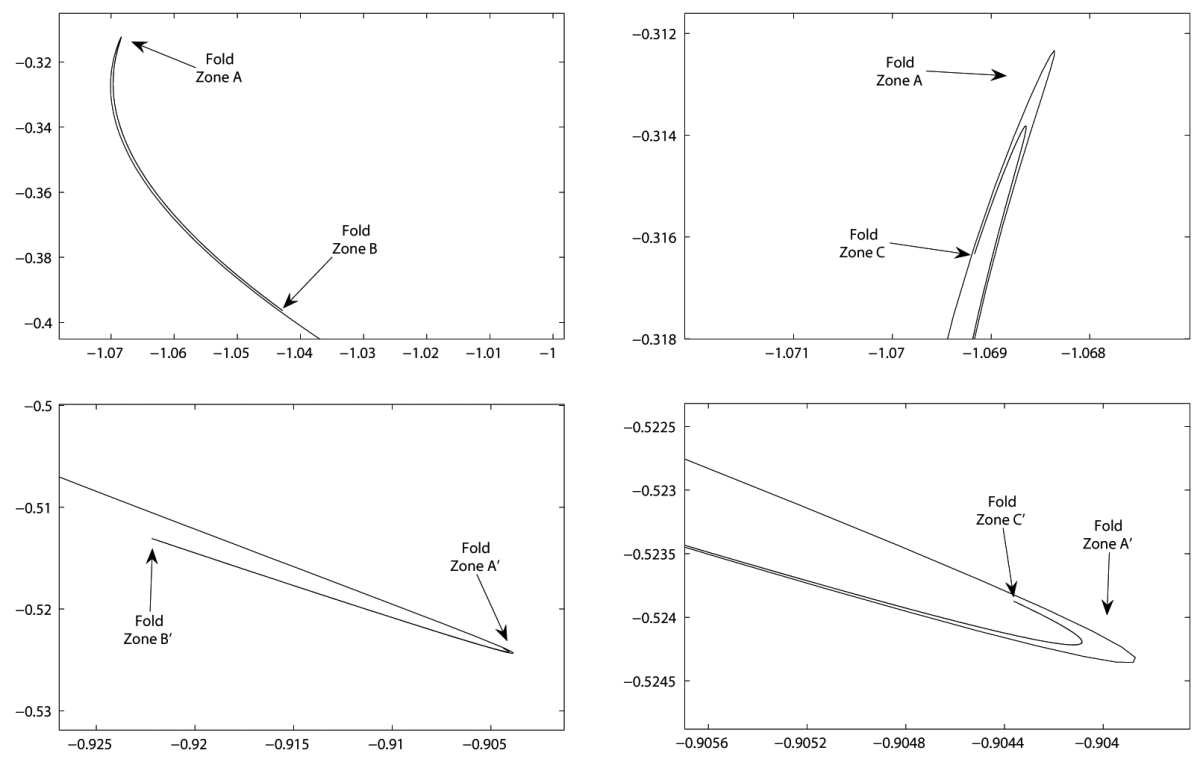

Figure 9: Details of the unstable manifold for $A=0.71$. 

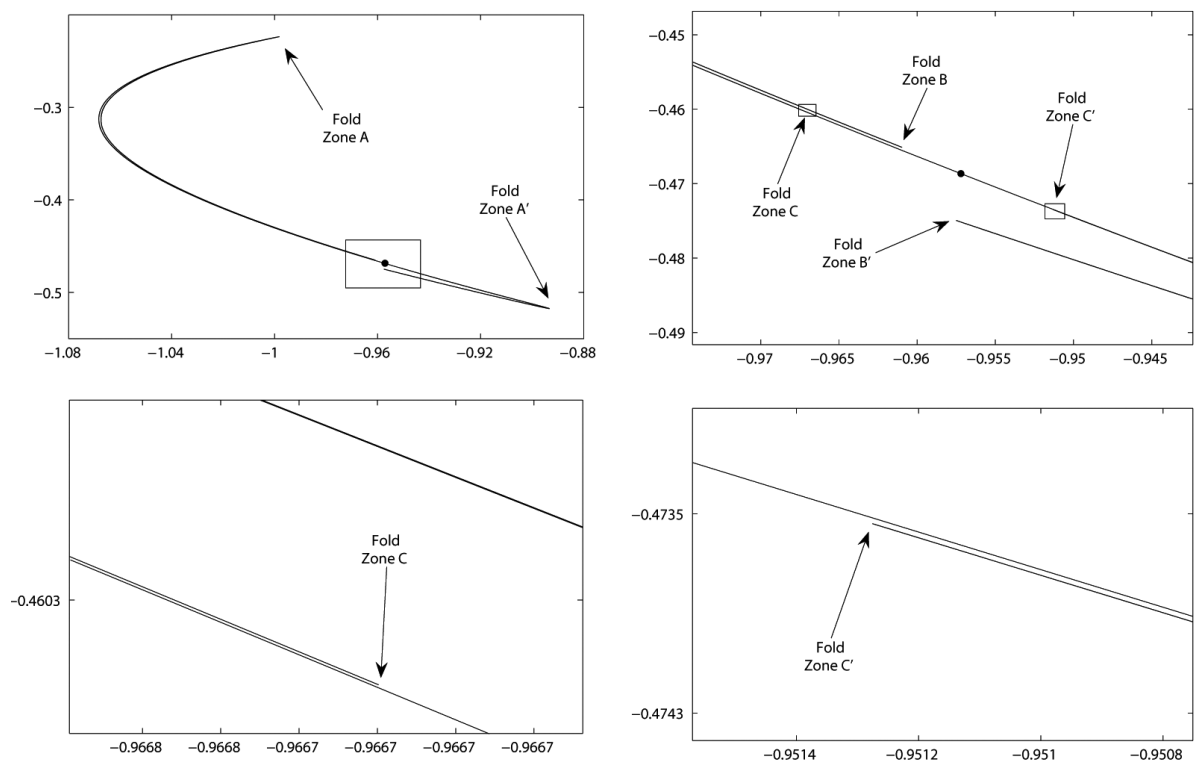

Figure 10: Unstable manifold for $A=0.735$. The SFP is marked with a black circle.
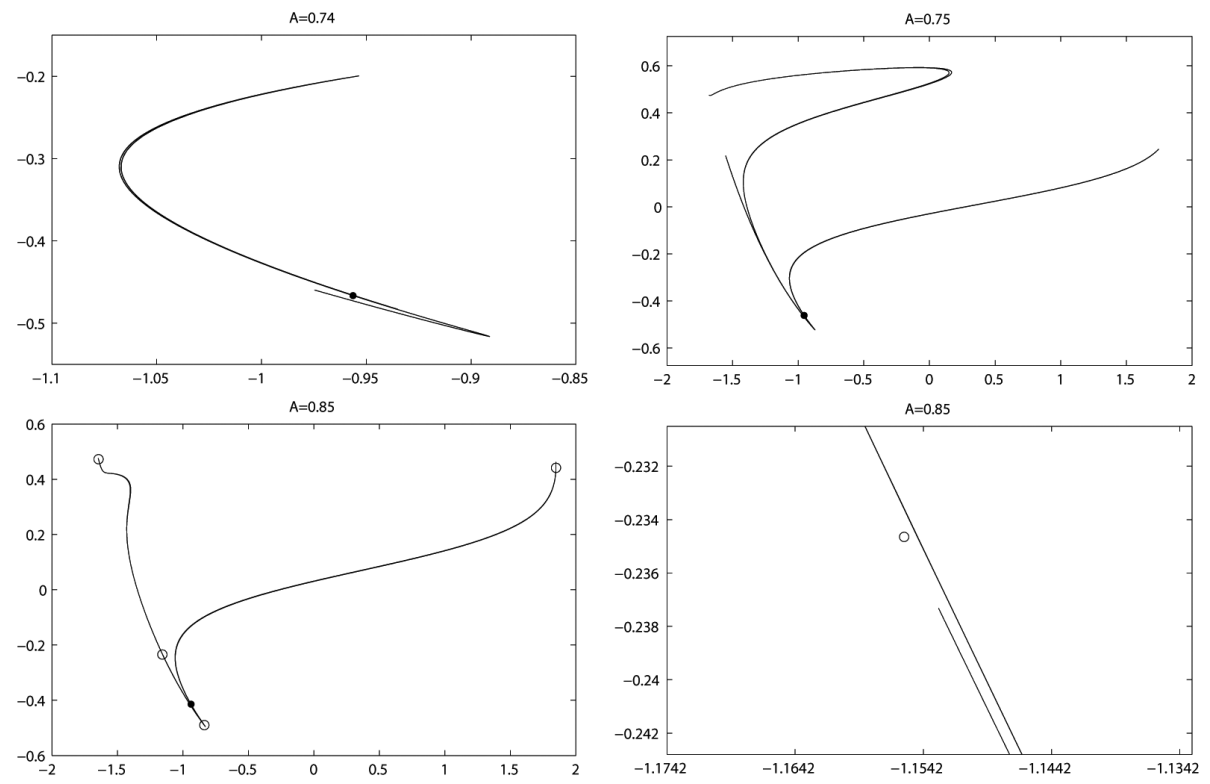

Figure 11: Unstable manifolds for $A=0.74, A=0.75$, and $A=0.85$. The SFP is marked with a black circle. For $A=0.85$, the four attracting periodic points (marked with circles) are near the main fold zones. 

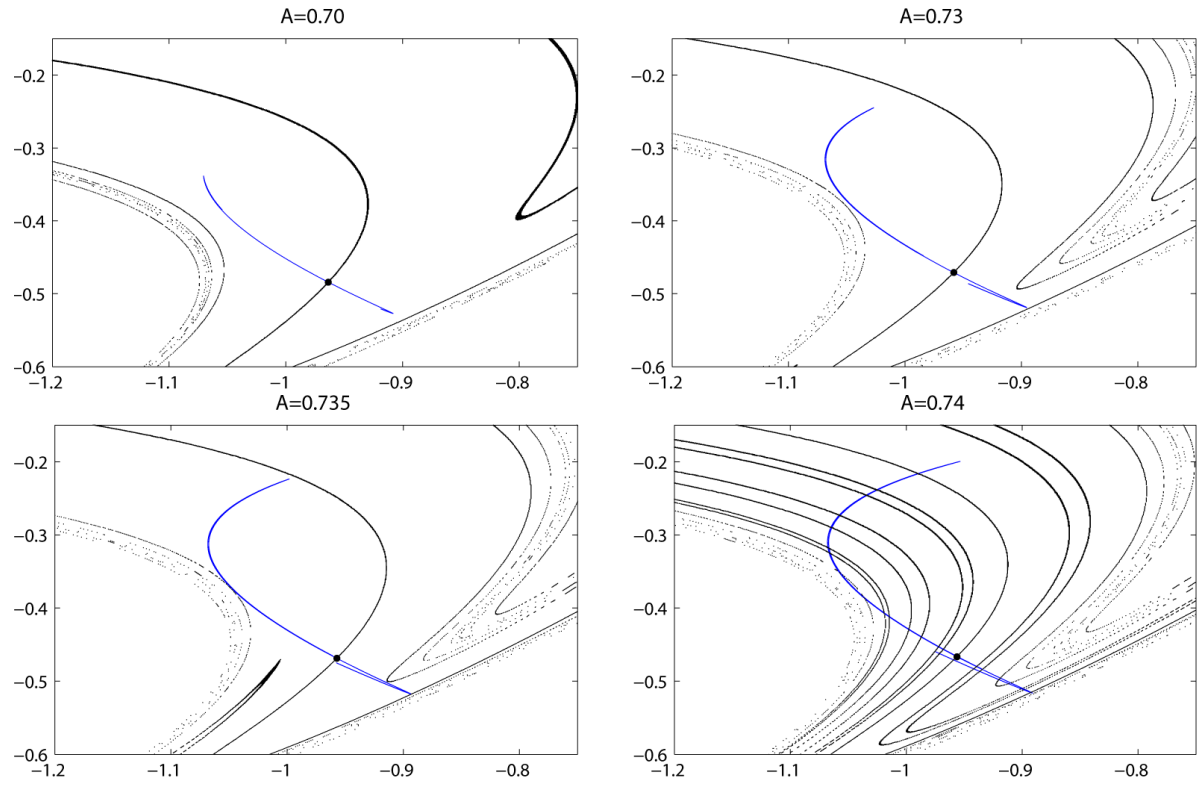

Figure 12: Stable (black) and unstable (blue) manifolds for $A=0.70, A=0.73$, $A=0.735, A=0.74$. The SFP is marked with a black circle.

These horseshoes are "quadruple" since each branch of each invariant manifold intersects the two branches of the other manifold.

For $A=0.74$ the first set of horseshoes has been already created (Fig. 12), and for $A \approx 0.748$ occurs the sudden expansion of the size of the attractor, causing new intersections between the stable and unstable manifolds and new horseshoes (Fig. 13, $A=0.75$ ). From this point, chaotic and non-chaotic zones alternate, because homoclinic tangencies between the stable and unstable manifolds are not continuously formed. For example, for $A \approx 0.785$ the chaos disappears because there are no homoclinic tangencies (Fig. $13, A=0.80$ ). This non-chaotic zone (Fig. 13, $A=0.85, A=1.05$ ) remains until $A \approx 1.11$ (Fig. 14 $A=1.10, A=1.15$ ), when new homoclinic tangencies are formed. This alternation of chaotic and nonchaotic zones is maintained until $A \approx 1.2835$ (Fig. 14. $A=1.21$ for a non-chaotic zone and $A=1.25$ for a chaotic zone).

For $A \approx 1.2835$ the last set of horseshoes is undone (Fig. 15), in a process inverse to the formation of horseshoes for $A \approx 0.735$. In fact, for these two values of $A$, the invariant manifolds are qualitatively similars.

Finally, for $1.2835 \lesssim A \lesssim 1.72$ there is not any horseshoe. Moreover, there are not homoclinic tangencies, and therefore there is no chaos. 

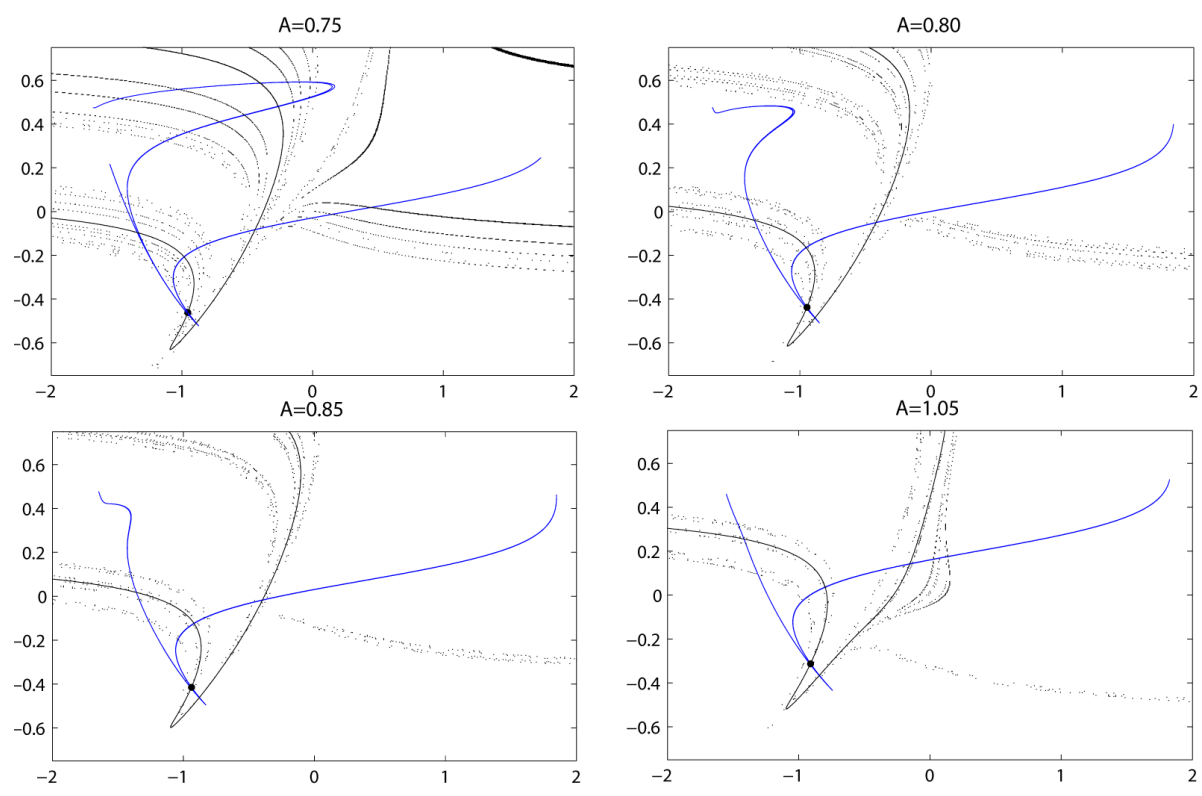

Figure 13: Stable (black) and unstable (blue) manifolds for $A=0.75, A=0.80$, $A=0.85, A=1.05$. The SFP is marked with a black circle.
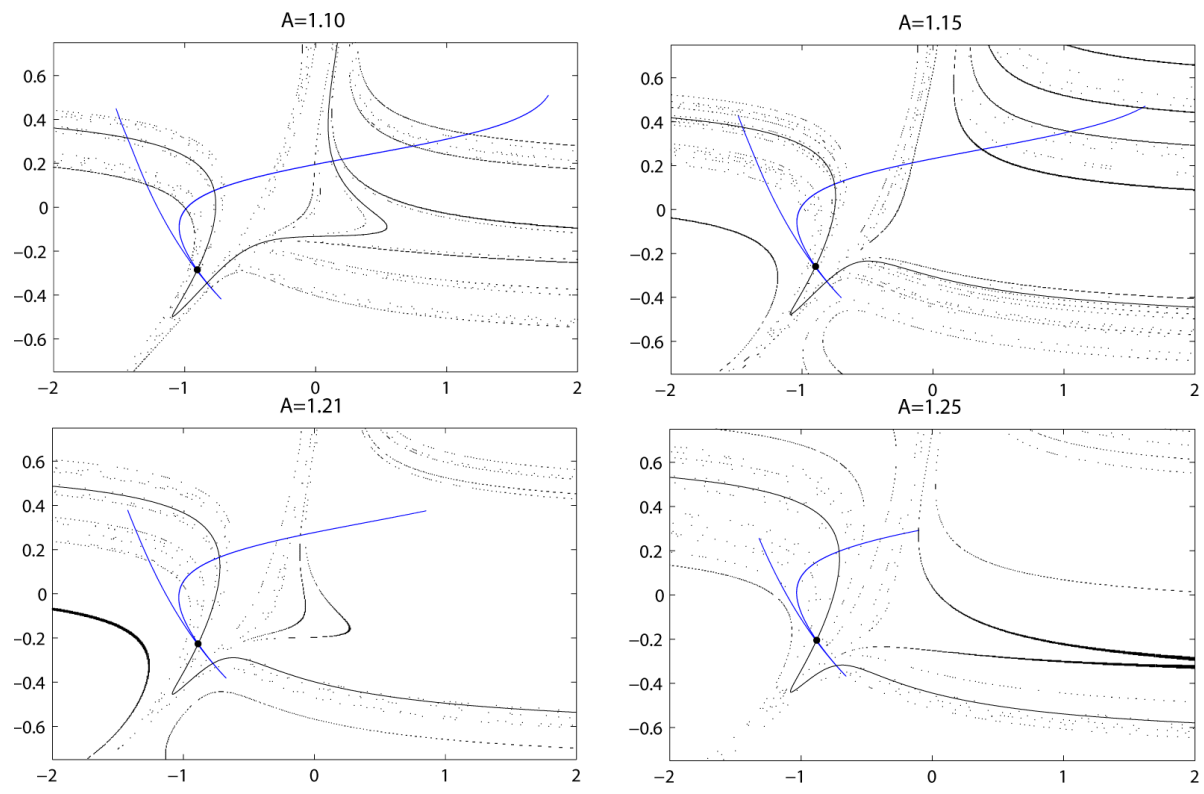

Figure 14: Stable (black) and unstable (blue) manifolds for $A=1.10, A=1.15$, $A=1.21, A=1.25$. The SFP is marked with a black circle. 

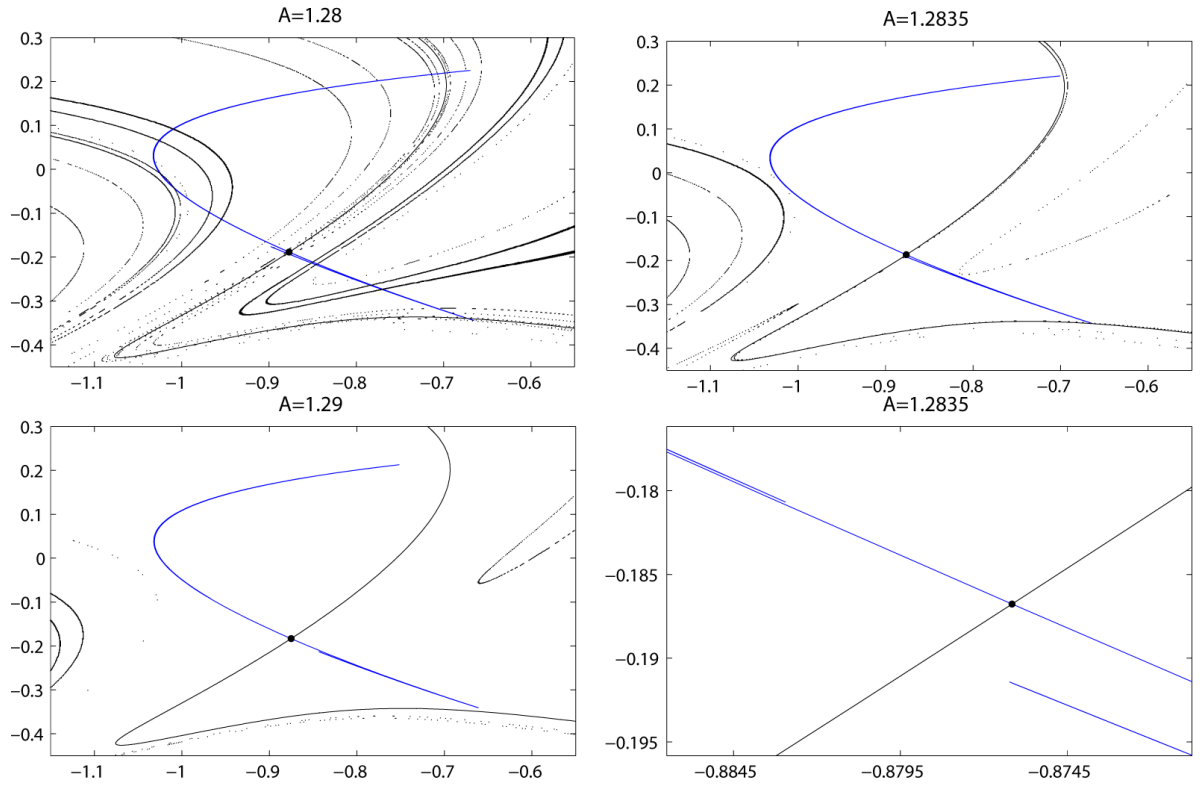

Figure 15: Stable (black) and unstable (blue) manifolds for $A=1.28, A=$ 1.2835, $A=1.29$. The SFP is marked with a black circle.

\section{Algorithms}

- The Poincaré map, $f$, is computed solving the system (1) using a RungeKutta method of order 4-5, with a local error of $10^{-12}$.

- The SFP is obtained with an absolute error of $10^{-12}$, using a method, that we shall describe below, based on the fact that the iteration of a neighbourhood of the SFP is also a neighbourhood of the SFP.

This algorithm is as follows: first consider an initial mesh $M_{0}$ of a square interval $I_{0}=\left[x_{0}^{1}, x_{0}^{2}\right] \times\left[y_{0}^{1}, y_{0}^{2}\right]$ containing a unique $\operatorname{SFP}\left(x^{*}, y^{*}\right)$.

Then, given a positive $\epsilon_{0}$, we consider the set

$$
C_{1}=\left\{(x, y) \in M_{0}:\left\|f(x, y)-\left(x^{*}, y^{*}\right)\right\|<\epsilon_{0}\right\} .
$$

Next we repeat this process with another mesh $M_{1}$ of the square interval $I_{1}=\left[x_{1}^{1}, x_{1}^{2}\right] \times\left[y_{1}^{1}, y_{1}^{2}\right]$, where

$$
\begin{array}{ll}
x_{1}^{1}:=\min \left\{x:(x, y) \in C_{1}\right\} & ; x_{1}^{2}:=\max \left\{x:(x, y) \in C_{1}\right\} \\
y_{1}^{1}:=\min \left\{y:(x, y) \in C_{1}\right\} & ; y_{1}^{2}:=\max \left\{y:(x, y) \in C_{1}\right\},
\end{array}
$$

and for another positive $\epsilon_{1}<\epsilon_{0}$. Obviously $I_{1} \subseteq I_{0}$.

Thus, repeating this process, with a suitable choice of meshes $M_{n}$ and $\epsilon_{n}$, we can obtain a sequence of nested squared intervals $I_{0} \supset I_{1} \supset I_{2} \ldots$ such 
that

$$
\left(x^{*}, y^{*}\right)=\bigcap_{n=0}^{+\infty} I_{n} .
$$

For example, in our case, we have chosen the initial square interval $I_{0}=$ $[-1.25,-0.25] \times[-0.6,0.4]$ and an initial mesh $M_{0}$ of $60 \times 60$ points for every $A$, with $\epsilon_{0}=10^{-1}$. In the next iterations we have considered meshes of $30 \times 30$ points, and $\epsilon_{n}=10^{-n-1}$. We have to note that, in this case, it is not necessary to use other methods based on model perturbations, as the control methods of OGY or Pyragas (see [9, 10, 11).

- In order to compute the invariant manifolds, first we estimate their tangential slopes at the SFP. This is done by using the eigenvectors of the Jacobian matrix $D f$ of the Poincaré map at the SFP.

- To compute the unstable manifold we iterate a segment with length of order $10^{-3}$, centered at the SFP, with the appropriate slope previously found. The number of iterations may change with the value of $A$, but it is usually between 12 and 24 .

- For the stable manifold, two complementary methods have been used.

- First, we have iterated twice a small segment (with the appropiate slope), using the inverse of the Poincaré map. As was pointed out in Section 3.1. this "inverse method" is only valid to compute the branches of the stable manifold until they are out of range. So the inital segment must be small enough to keep the iterations within range.

- Next, we find the points of a mesh such that, after $n$ iterations, their distance to the SFP is less than a given $\epsilon$. In our case we used $n=8$, a mesh of $900 \times 900$ points, and $10^{-3}<\epsilon<10^{-2}$.

\section{Final remarks}

The main results obtained are the following:

- The existence of Smale horseshoes in the forced BvP oscillator (1) has been explicitly checked, depending on the amplitude of the external forcement $A$. In particular, the horseshoes appear for $A \in(0.735,1.2835)$, approximately.

- The chaotic zones of the bifurcation diagram are related to creation and/or destruction of horseshoes, i.e., to the existence of homoclinic tangencies between the invariant manifolds.

- The sudden expansion of the attractor seems to be related to the creation of the first set of horseshoes. 


\section{Acknowledgments}

We would like to thank José A. Rodríguez, from the University of Oviedo for his valuable help. Both authors were partially supported by Junta de Extremadura, and MCYT-FEDER Grant Number MTM2004-06226.

\section{References}

[1] S. Rajasekar. Dynamical structure functions at critical bifurcations in a Bonhoeffer-van der Pol equation. Chaos, Solitons and Fractals 7 (11) (1996), 1799-1805.

[2] A. C. Scott. Neurophysics. Wiley, New York (1977).

[3] S. Rajasekar, S. Parthasarathy, M. Lakshmanan Prediction of horseshoe chaos in BVP and DVP oscillators. Chaos, Solitons and Fractals 2 (3) (1992), 271-280.

[4] J. Guckenheimer, P. Holmes. Nonlinear oscillations, dynamical systems, and bifurcations of vector fields. Spinger-Verlag New York (1986)

[5] W. Wang. Bifurcations and chaos of the Bonhoeffer-van der Pol model, J. Phys. A: Math. Gen. 22 (1989), L627-L632.

[6] J. P. England, B. Krauskopf, H. M. Osinga, Computing one-dimensional stable manifolds and stable sets of planar maps without the inverse, SIAM Journal of Applied Dynamical Systems 3 (2) (2004), 161-190.

[7] D. Hobson, An efficient method for computing invariant manifolds of planar maps, Journal of Computational Physics 104 (1991), 14-22.

[8] E. J. Kostelich, J. A. Yorke, Z. You, Plotting stable manifolds: error estimates and noninvertible maps, Physica D 92 (1996), 210-222.

[9] E. Ott, C. Grebogi, J. Yorke. Controlling Chaos. Phys. Rev. Lett. 66 (1990), 1196-1199.

[10] S. Rajasekar. Controlling Unstable Periodic Orbits in a Bonhoeffer-van der Pol equation. Chaos, Solitons and Fractals 5 (1995), 2135-2144.

[11] M. Ramesh, S. Narayanan. Chaos control of Bonhoeffer-van der Pol oscilaltor using neural networks. Chaos, Solitons and Fractals 12 (2001), 2395-2405. 